\title{
Size-distance judgments with random letter stereograms'
}

\author{
PHILIP TOLIN ${ }^{2}$ \\ UNIVERSITY OF IOWA
}

\begin{abstract}
Using random letter stereograms, the size-distance relationship was investigated in the absence of a number of distance cues that are typically present when objects and drawings are used as stimuli. With depth differences signaled only by differences in binocular disparity, Ss reported that the apparently farther of two equal-sized stereoscopic images appeared larger as predicted by the size-distance invariance hypothesis. However, distance judgments of equally displaced figures were largely independent of relative size variations. Order of report of relative size and relative distance, figure placement with respect to the ground, and relative matrix densities of figures and ground did not reliably affect judgments.
\end{abstract}

Stereograms of the random-dot (Julesz, 1960, 1963) and random-letter (Kaufman, 1964a, 1964b, 1965) varieties have provided a useful technique for investigating a number of problems in binocular vision. These materials, which eliminate such possible depth cues as object familiarity, texture gradients, double images, and monocularly viewed contours, consist of two monocularly presented matrices of randomly placed dots or letters. The matrices are virtually identical, the sole difference being a horizontal shift of one or more portions of the matrix viewed by one eye relative to the corresponding portion of the other eye. The resultant binocular disparity produces the impression of a figure located at a depth different from that of the ground. The physical correlate of apparent figure size is the area of the displaced portion of the field. The correlate of apparent depth is the amount of horizontal displacement.

Using random-letter stereograms with binocular figures appearing in front of the ground (caused by displacement in a nasal direction), Bunge and Bechtoldt (1967) found that with the visual angles of two figures held constant, the figure judged to be farther from $\mathbf{S}$ was also judged larger, as predicted by the notion of size constancy. On the other hand, when two figures of different apparent size were equally displaced, judgments of relative depth were independent of variations in relative size, despite the assertion by Carlson and Tassone (1962) that Ss make an assumption that the apparent size of an object diminishes with increasing distance. The present research represented a replication and extension of the Bunge and Bechtoldt study. As previously reported, these authors used stimuli in which two figures were presented in front of a ground. Of interest in the present research was the problem of whether the size-distance functions obtained when the figures are located behind the ground are comparable to those obtained when the figures are located in front of the ground.

In the Bunge and Bechtoldt research, the figures could not be seen monocularly since the figures had a composition (matrix density) similar to that of the ground. By making the figure areas of the stereograms more dense than the area representing the ground, it is possible to create figures that can be seen monocularly. A second question of interest in the present research involved the extent to which elimination of monocular cues for form identification affects distance judgments when relative figure size is varied.

Finally, Bunge and Bechtoldt obtained evidence suggesting that size judgments were affected by the order in which size and distance judgments were made. Although the authors attributed this to a sampling error, additional data bearing on the effect of order of report were collected in the present study.

\section{METHOD}

\section{Stimulus Materials}

The stimulus materials were random-letter stereograms generated by an IBM 7044 computer and printed by an IBM 1401 line printer. The computer was programmed to assign either an upper-case letter or a blank to each cell of a 72 by 90 matrix. In all cases, the areas designated as figures, i.e., those portions of the matrices that were shifted, had a matrix density, expressed in terms of the percentage of filled cells, of $60 \%$. The matrix density of the ground was either $60 \%$ (no monocularly visible figures) or $20 \%$ (monocularly visible figures). The computer outputs were photographed, from a height of $21 \mathrm{in.}$, as $35-\mathrm{mm}$ fine-grain Kodak positives from high-contrast negative film. The slides were mounted with $63 \mathrm{~mm}$ separation and presented in a rigid but adjustable Realist Model 2062 electric stereoviewer within $1 / 2$ in. of S's eyes. The light intensity of the viewer (without slides) was $3.54 \mathrm{ft}-\mathrm{c}$, as measured by a Weston Model 756 light meter. The stereograms subtended a visual angle of $23.26 \mathrm{deg}$ and extended over nearly the entire visual field of the stereoviewer.

The stimuli consisted of two square figures placed within diagonally opposed quadrants of a square ground. Each figure took one of the following four sizes: $15 \times 19,16 \times 20,17 \times 21$, or $18 \times 23$ (rows $\times$ columns), but since letter height was greater than letter width, the figures were square. The relative sizes of the two figures, expressed in terms of areal ratio (ratio of area of left figure to area of right figure), were $1.29: 1,1.11: 1,1: 1,1: 1.11$, and $1: 1.29$. The depths of the figures relative to the ground were varied by introducing horizontal shifts of from one to three columns (15.5 min to $46.5 \mathrm{~min}$ of visual angle) of the figure presented to one eye relative to the corresponding figure presented to the other eye. Increasingly large shifts in the nasal direction caused the fused image to appear farther in front of the ground; increasingly large shifts in the temporal direction caused the image to appear farther behind the ground. The notation used in this report signifies that the left (L) or right (R) figure appeared behind the other figure by an amount determined by a differential shift of one or two columns $(L 2, L 1, R 1, R 2)$ or that the two figures were located at the same depth relative to the ground $(L=R)$ at each of the depth levels used.

\section{Procedure}

Chair-height and chin-rest adjustments were first made to maximize S's comfort. A series of six slides was then presented to aid in the adjustment of the focus and interocular distance of the stereoviewer and to determine whether $S$ was able to identify correctly relative depths of stereoscopically viewed objects. Those Ss who were unable to correctly identify displaced figures with minimal coaching were dismissed. Instructions acquainting $S$ with the nature of stereograms, figure location, and the appropriate responses, were then read. The Ss were told that the two figures would always differ in both size and depth and that they were to indicate which was farther away and which was larger. "Doubtful" responses were not allowed.

Each $S$ was then given a training sequence of 12 stereograms, 6 containing binocular figures having large depth differences and 6 


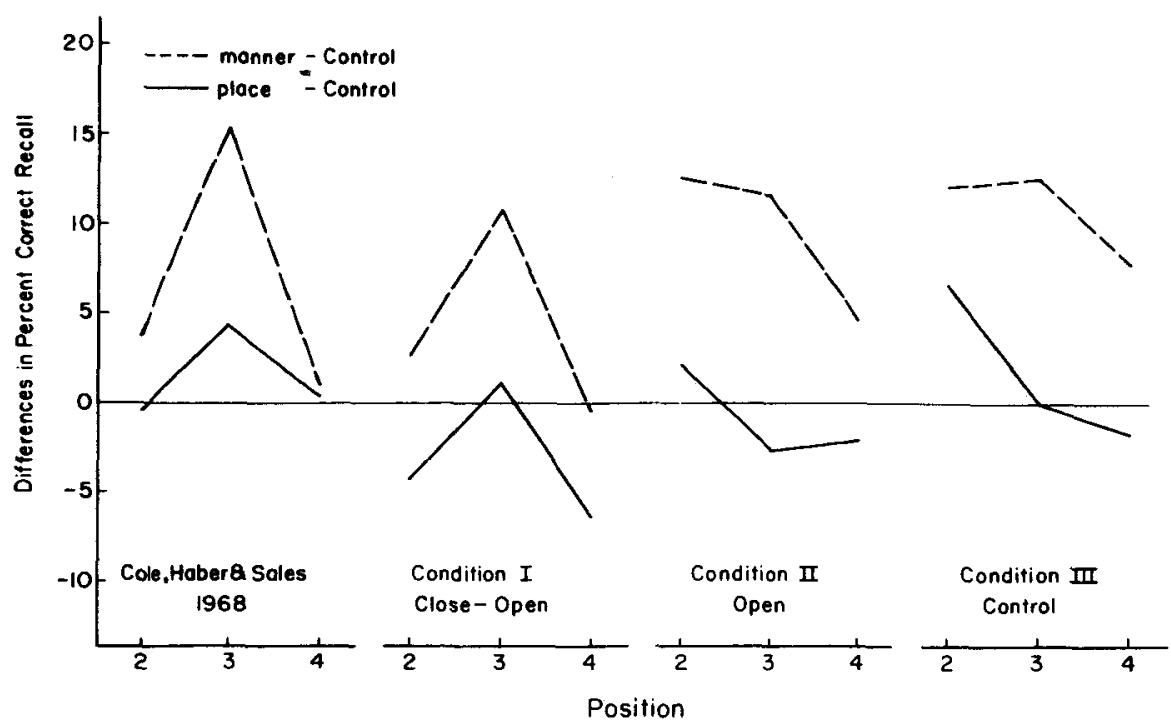

EXPERIMENT 2

\section{Subjects}

Twenty-six University of California undergraduate students served as volunteer Ss. They were tested individually in a session lasting approximately $1 \mathrm{~h}$.

\section{Stimuli}

The same stimuli were used as in Experiment 1. Sounds were presented at the rate of $1 / \mathrm{sec}$ to the beat of a metronome. Since only sequences with a similar manner of articulation have been shown to differ from controls, place-of-articulation sequences were excluded from this experiment. Seventy-two sequences of three sounds each were used: 12 sequences for each of the different manner-of-articulation sequences (voiced, unvoiced, and nasal), and 36 control sequences. Thus, each sound occurred in each position four times in the manner-of-articulation sequences and four times in the control sequences. Stimuli were balanced over positions and no sound was repeated in a sequence. Each $S$ received the 72 sequences in the same order, which was a random selection of sequences.

To manipulate rehearsal processes, $S$ s were required to trace a drawing on a toy known as "Etch-a-Sketch." This is a rectangular object with a screen on its surface, measuring $7 \frac{1}{4} \times 5 \frac{1}{4}$ in. Drawings of objects (e.g., a house) or geometric designs on plastic plates were placed directly over the screen. Two knobs on the bottom right- and left-hand corners of the Etch-a-Sketch manipulated a pointer in either the horizontal or vertical direction, so that by manipulating both knobs separately or together $S$ could trace the picture on the plastic plates. The designs on the plates were drawn with a $1 / 4$-in. felt pen, so that $S$ could see through the outlines of the design as he traced.

\section{Procedure}

Each S heard the following instructions: "This is an experiment in spontaneous thought. We are studying what determines how thoughts simply pop into consciousness. You will be hearing three randomly ordered sounds at a time from the following nine. Please repeat these after me: $\mathrm{Ba}, \mathrm{Pa}, \mathrm{NGa}, \mathrm{Ma}, \mathrm{Ta}$, $\mathrm{Na}, \mathrm{Ka}, \mathrm{Da}, \mathrm{Ga}$.

"The experiment will be run as follows: First you will hear three sounds said to the beat of a metronome, followed immediately by the signal, 'Start.' Simply let the sounds register-do not try and remember them or repeat them. At the word, 'Start,' trace the figure on the Etch-a-Sketch in front of you. After $8 \mathrm{sec}$, a voice will say, 'Report.' Stop tracing immediately and say the first three sounds which come into your mind.

"You can see how we will be studying spontaneous thought from a specific set of sounds. It is necessary to ask you to help us in this respect. In order to make sure the sounds you say are spontaneous, you must keep a blank mind during the task. We know that it is possible to keep a blank mind, but it requires some practice, so we will have a series of practice trials. We are interested only in the first three sounds that come to mind, whether or not they bear any relation to the three sounds you have heard.

"You have a $25 \%$ chance of winning $\$ 5.00$ in this experiment. For each figure that you trace without any mistakes you will win 10 points. If you end the sketch in the bottom left-hand corner - the start - you will get a bonus of 2 points. Each time that you go out of the line you lose 1 point. If you start before the signal or stop late you lose 2 points. As soon as you are finished with one sketch go immediately to the next one. Thus, those subjects who finish the most sketches most accurately will win $\$ 5.00$.

"Are there any questions? We will now have the 10 practice trials. Remember to simply let the sounds register."

Ss were then given the 10 practice trials. After 5 trials, E stopped the procedure and asked $S$ if he was having any trouble. Since it was found, during pretesting, that Ss were attending to the Etch-a-Sketch, and were able to hear the sounds without repeating them, Ss were not quizzed about rehearsing until after the experiment. All Ss reported that they did not rehearse the sounds.

Ss then heard the 72 sequences of sounds. E recorded the Ss' reports on each trial. As each tracing was completed, E scored the tracing, cleared the screen on the Etch-a-Sketch, replaced the pointer to start, and returned the apparatus with a new design to the S. Since two Etch-a-Sketch boards were used, $S$ always had a new tracing to begin after finishing one.

\section{Scoring}

For the basic analysis, a sound was scored as correct only if $S$ named the proper sound in the proper position. For a second analysis, a sound was scored as correct if it was recalled in any of the three positions.

\section{Results}

In order to determine if sounds were kept in memory 


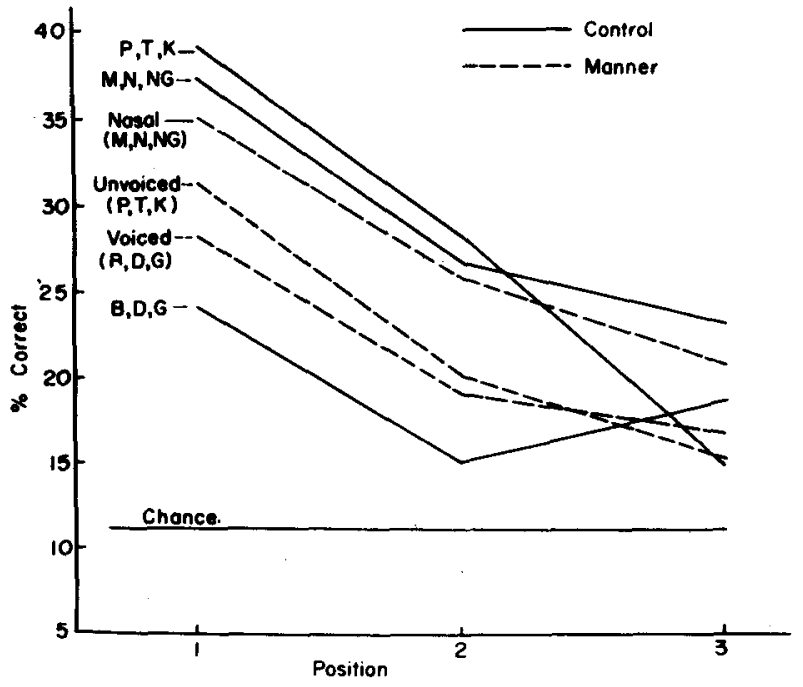

Fig. 3. Per cent correct recall for the three sounds in the manner-of-articulation sequences, and in three matched control sequences, as a function of position. Data are summed over $30 \mathrm{Ss}$.

throughout the procedure on each trial, the per cent correct recall was scored for sounds in each of the manner and control sequences for the three positions, and these data are shown in Fig. 3. For this analysis, a sound was scored as correct only if it was recalled in the presented position. $T$ tests showed that sounds were significantly easier to recall in Position 1 than in Positions 2 or $3(\mathrm{p}<.01)$, although the latter two positions do not differ from each other. $T$ tests performed on these data show that sounds in all three positions were recalled significantly better than by chance $(p<.01)$.

An identical analysis was performed when a sound was scored as correct if it was recalled in any of the three positions. Again, Position 1 was easiest to recall, and performance was greater than chance for all three positions.

To determine the effects of similarity on recall, each sound in each position in the manner-of-articulation sequences was compared to the same sound in the respective position in the control sequences. The percentage correct for each sound was determined and then subtracted from the comparable score in the control sequences. Thus, in Fig. 4 , for a particular sound in a manner-of-articulation sequence, a positive number indicates that the sound was confused more of ten when in a context of similar manner of articulation and a negative number indicates that sounds were confused less often in manner than in control sequences. The scores for the three sounds defining each condition (e.g., Ba, Da, $\mathrm{Ga}$ for voiced) were combined and are shown in Fig. 4. $T$ tests showed that for any position, there was no significant difference between the number of times a sound was confused when in a similar feature sequence than when the sound was in a control sequence.

Briefly summarizing these results, it appears that when $S$ does not attend to his rehearsal, while he still can recall the items with greater-than-chance accuracy, the pattern of errors no longer is predictable by the distinctive feature differences that worked for more conventional testing situations.

\section{Subjects}

\section{EXPERIMENT 3}

Sixty undergraduate University of California students served as Ss. They received 2 points on their final exam for participating in the experiment. They were tested individually in a session lasting $1 / 2 \mathrm{~h}$.

\section{Stimuli}

A series of sequences of five consonants, each of which was paired with the vowel /a/, were selected from among the nine consonants in Fig. 1. They were presented by a Wollensak tape recorder to the beat of a metronome to each $S$ seated in a room with $\mathrm{E}$. The sounds were recorded so that they were heard at a rate of $3.3 / \mathrm{sec}$. Four different types of sequences were used, differing according to the rules of selection of the items from Fig. 1. In the three different manner-of-articulation sequences, the second, third, and fourth sound in each sequence was the same manner of articulation. The sounds on either side of the sequence had neither place nor manner similar to the adjacent sound. Such a sequence for voiced consonants would be $\mathrm{Ta}, \mathrm{Ba}$, $\mathrm{Da}, \mathrm{Ga}, \mathrm{Na}$. In the control condition, no sound in any of the positions had either place or manner similar to adjacent sounds. Such a sequence would be $\mathrm{Ba}, \mathrm{Ta}, \mathrm{Ga}, \mathrm{Na}, \mathrm{Pa}$. For each of the three manner-of-articulation conditions, six sequences of sounds were used, so that each sound appeared in each position twice for each manner of articulation. There were 18 control sequences, so that each sound appeared in each position six times. Thus, each $S$

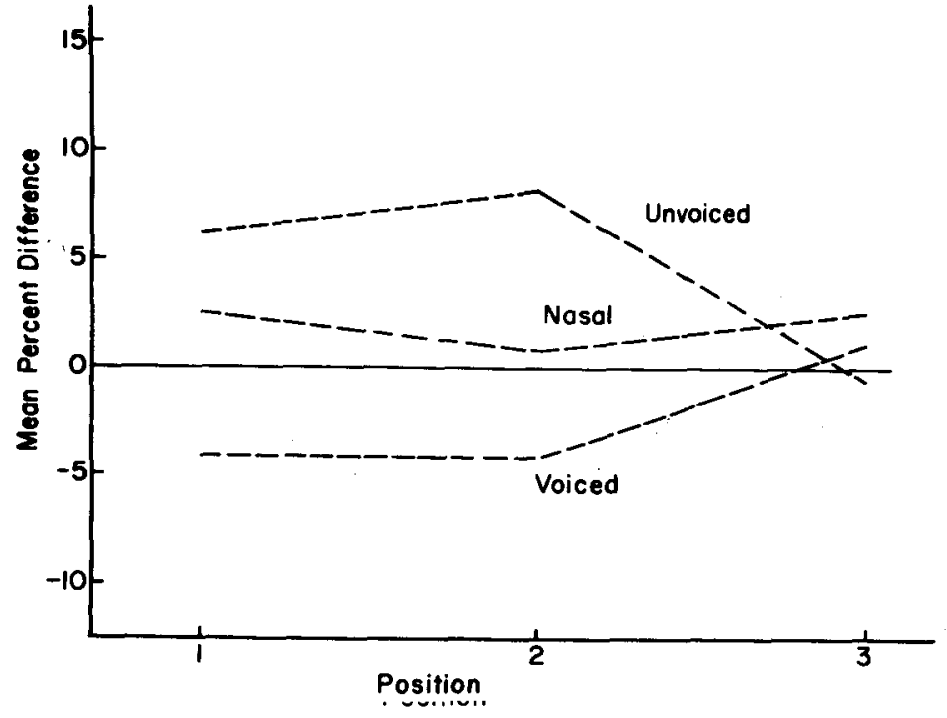

Fig. 4. Differences in per cent correct recall between sounds in each of the manner-of-articulation sequences and in the control sequences as a function of position. Data are summed over $30 \mathrm{Ss}$. 


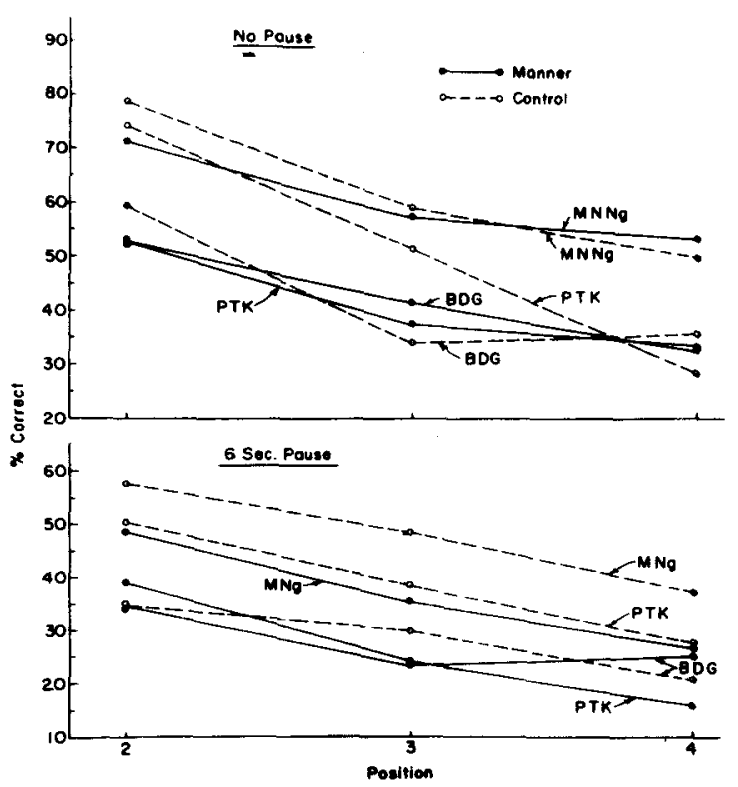

Fig. 5. Per cent correct recall for the three sounds in the manner-of-articulation sequences and the control sequences as a function of position for the 6-sec pause and for immediate recall.

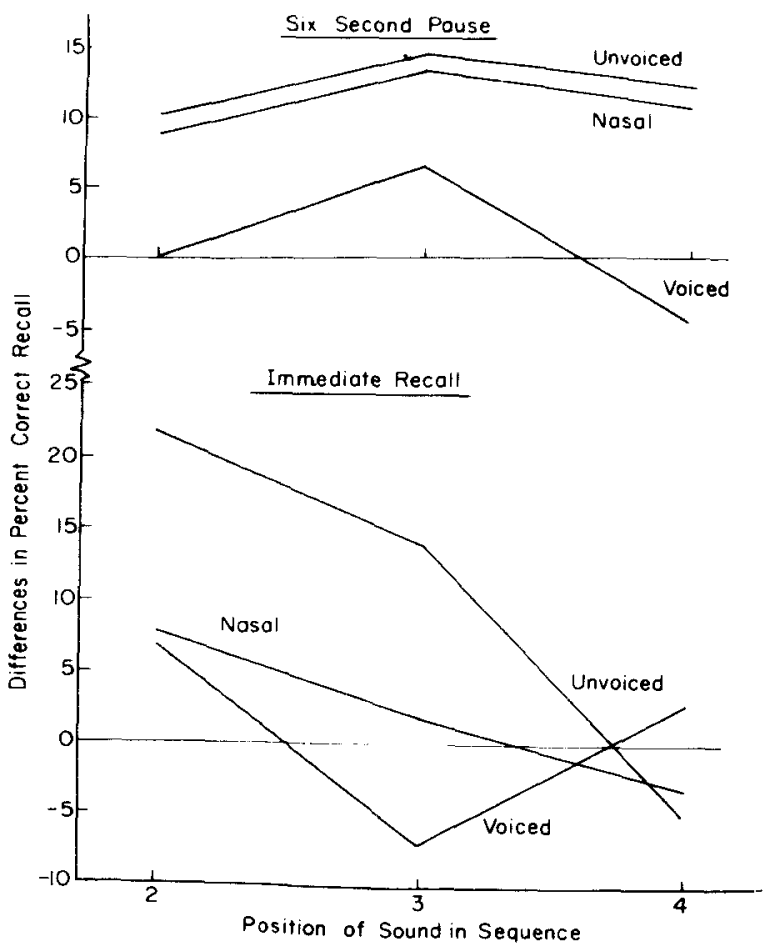

Fig. 6. Differences in per cent correct recall between sounds in each of the manner-of-articulation and the control sequences as a function of position for the 6-sec pause and for immediate recall.

heard 36 sequences. Within each type of sequence, sounds were balanced over positions. No sound was repeated in a sequence. Each $S$ received the 36 sequences in the same order.

\section{Procedure}

All instructions were heard via the tape recording. The S was told that he would hear a list of five sounds presented rapidly in time to a metronome.

Group 1: Ss were told that starting on the beat following the last sound, they must repeat back the sounds in the correct order in time to the metronome.

Group 2: Ss were told that following the last sound, there would be a pause of $6 \mathrm{sec}$, and they then would hear the signal, "Okay." On the next beat of the metronome, following this signal, they were told to report back the sounds in the correct order in time to the metronome.

\section{Scoring}

For the basic analysis, the $\mathrm{S}$ was scored as correct for each sound in a sequence only if he named the proper sound in the proper position.

\section{Results}

Figure 5 shows that accuracy declines for all sounds and sequences as a function of position, and that the differences between the two pause conditions is also significant. Figure 6 shows the respective differences between the three manner sequences from the control sequence for the 6-sec pause and the immediate recall groups. Unvoiced and nasal sequences are still more difficult than control sequences. The voiced sequences become less differentiated from the control sequences except for the middle position. Thus, even with the much higher rate of presentation, requiring a $6-\mathrm{sec}$ pause produces, by and large, the same pattern of errors as found with slower rates and a pause.

When immediate recall is required, only the unvoiced features confuse significantly more than the control sequences and only for the second and third positions. Thus, in general, immediate recall improves performance and especially reduces confusions among potentially confusable distinctive features.

\section{DISCUSSION}

In Experiment 1, when articulation was prevented, the basic finding of Cole, Haber, and Sales (1968) was replicated. Prevention of gross articulatory movements (by asking $S$ to hold his mouth open) did not affect the distinctive feature encoding of consonant sounds. This finding seems to rule out the importance of gross articulatory movements for the encoding of consonant sounds in a serial recall task when the vocalic environment is constant.

In Experiment 2, our prediction that rehearsal could be blocked without knocking out all short-term memory was confirmed. When rehearsal is presumed to be blocked or minimized using the present procedure, sounds sharing a feature of manner of articulation are not confused more of ten than sounds not sharing this feature. Thus, the distinctive feature substitution observed in Experiment 1 in manner sequences can be eliminated. This suggests that rehearsal is at least one place where distinctive feature encoding occurs.

In the third study, the importance of distinctive features governing encoding was shown to be dependent upon a pause between fast presentation and recall. Without such a pause, apparently recall can be made without normal rehearsal, perhaps from some image.

In the first experiment, Ss who were unable to articulate still reported that they were able to "hear" themselves rehearsing quite easily, although they experienced some difficulty in the practice trials. This suggests that Ss normally articulate sounds and at the same time "hear" themselves saying the sounds. When the articulatory movements are prevented in the way done here, it may be that the alternate mechanism of "hearing" the sounds during rehearsal still occurs and is completely adequate. When both processes are blocked, as in Experiments 2 and 3, encoding according to these features is altered. 
While articulatory movements do not appear important in distinctive feature encoding, an articulatorily based code is not entirely ruled out; feedback may persist even from blocked movements. Further, it is possible that neural impulses that represent articulatory movements are paired with certain sounds and are so overlearned that the actual articulation of the sound is not needed to instigate the impulse. Thus, neural impulses may once have been paired with the articulation and subsequent acoustic result of the sound, but now either hearing the sound or the articulation of the sound may converge on the same central encoding mechanism.

\section{REFERENCES}

BLUM, G. S. A model of the mind New York: Wiley, 1961.

COLE, R. A., HABER, R. N., \& SALES, B. D. Mechanisms of aural encoding: 1. Distinctive features for consonants. Perception \& Psychophysics, 1968, 3, 281-284.

HINTZMAN, D. L. Articulatory coding in short-term memory. Journal of
Verbal Learning \& Verbal Behavior, 1967, 6, 312-316.

LIBERMAN, A. M. Some results of research on speech perception. Journal of the Acoustical Society of America, 1957, 29, 117-123.

WICKELGREN, W. A. Distinctive features and errors in short-term memory for English consonants. Journal of the Acoustical Society of America, 1966, 39, 388-398.

WICKELGREN, W. A. Auditory or articulatory coding in verbal short-term memory. Psychological Review, 1969, 76, in press.

\section{NOTES}

1. This research was supported in part by a grant from the United States Public Health Service, MH 10753, and a grant from the National Science Foundation, GB 5910, both to the third author at the University of Rochester. We would like to thank Willard Harley, Jr., for his suggestions, Gerald Blum for his comments on Experiment 2, and Jenifer Crook, Barbara Zeagel, and Patricia Becker for running Ss in these experiments.

2. Address: Department of Psychology, University of Rochester, River Campus Station, Rochester, New York 14627.

(Accepted for publication April 28, 1969.) 